\title{
Early intervention in psychosis: a map of clinical and research initiatives in Latin America
}

\section{Intervenção precoce em psicose: um mapa das iniciativas clínicas e de pesquisa na América Latina}

\author{
Elisa Brietzke ${ }^{1,2}$, Ary Gadelha Araripe Neto ${ }^{1,2,3}$, Álvaro Dias ${ }^{2}$, Rodrigo Barbachan Mansur ${ }^{1,2}$, Rodrigo Affon- \\ seca Bressan ${ }^{1,2,3}$ \\ ${ }^{1}$ Program for Recognition and Intervention in Individuals in At-Risk Mental State (PRISMA), Department of Psychiatry, Universidade Federal \\ de São Paulo, São Paulo, Brazil. \\ 2 Interdisciplinary Laboratory of Clinical Neurosciences (LINC), Department of Psychiatry, Universidade Federal de São Paulo, São Paulo, Brazil. \\ ${ }^{3}$ Program of Schizophrenia (PROESQ), Department of Psychiatry, Universidade Federal de São Paulo, São Paulo, Brazil.
}

\begin{abstract}
Objective: This article aims to review Latin America's early intervention services in psychosis and to shed light into their challenges and particularities. Method: An internet-based search comprising medical societies' websites, published articles, and major universities' websites was conducted and the results were critically discussed. Results: Latin American countries are profoundly deficient in specialized early intervention services. Our search found seven target services, four of which are based in urban areas of Brazil, inside tertiary hospitals or universities. Among the initiatives advanced by these centers, there are partnerships with the public educational system and other community-based efforts toward knowledge transfer. On the other hand, several challenges remain to be overcome, especially in relation to their expansion, which is necessary to match the existing demand.
\end{abstract}

Descriptors: Psychotic disorders; Early intervention; Disease prevention; Latin America; Mental Disorders

\section{Introduction}

Psychosis is a syndrome characterized by the presence of delusions and hallucinations, disintegration of thought processes, and blunted affect ${ }^{1}$, with schizophrenia being the prototypical psychotic disorder. Schizophrenia is currently believed to be caused by a combination of genetic and environmental factors, which establish complex interactions during brain development ${ }^{1,2}$. The natural history of schizophrenia usually involves a prodromal period, when mild symptoms progressively increase in severity, duration and frequency until crossing a threshold, then allowing a diagnosis to be established in light of current nosological classification systems ${ }^{3}$. This moment of diagnosis establishment is

\section{Resumo}

Objetivo: Este artigo tem o objetivo de revisar os serviços de intervenção precoce em psicose na América Latina e lançar luz sobre seus desafios e particularidades. Método: Foi realizada uma busca na internet compreendendo os websites de sociedades médicas e das principais universidades e artigos publicados por autores latino-americanos e os resultados foram discutidos criticamente. Resultados: Os países latino-americanos são profundamente deficientes no que diz respeito a serviços especializados de intervenção precoce em psicose. Nossa busca encontrou sete serviços principais, quatro dos quais baseados em áreas urbanas do Brasil, dentro de hospitais terciários ou universidades. Dentre as iniciativas promovidas por esses centros, há parcerias com o sistema público de educação e outros esforços baseados na comunidade para transferência de conhecimento. Não obstante, vários desafios ainda devem ser superados, relacionados sobretudo à expansão de tais serviços, necessária para abarcar a demanda existente.

Descritores: Transtornos psicóticos; Intervenção precoce; Prevençâo de doenças; América Latina; Transtornos Mentais called first episode and is considered to be an important milestone in illness evolution ${ }^{4}$.

In the majority of the cases, schizophrenia is associated with a devastating impact in the life of affected individuals, their families, and society ${ }^{5}$. Uncountable losses related to professional life, social relationships, scholar achievements, and economic burden associated with schizophrenia spectrum disorders have been considered as major reasons to develop endeavors to prevent, reduce severity, and rehabilitate individuals with psychosis ${ }^{6}$.

Several years of research in schizophrenia have shown that interventions taking place during the early stages of its course

\section{Correspondence}

Elisa Brietzke

Rua Machado Bittencourt, 222

Vila Clementino

São Paulo, Brazil

E-mail: elisabrietzke@hotmail.com 
have several advantages. They are possibly associated with less disruption in personal life, including unemployment, depression, substance abuse, and criminal, suicidal or aggressive behavior ${ }^{7-10}$. Conversely, it has been established that the duration of untreated psychosis (DUP) is related to unfavorable outcomes in the long run, including decreased response to antipsychotic treatment and higher risk of developing negative symptoms (for a meta-analysis, see: Perkins et al., 2005) ${ }^{11}$.

Early intervention is a clinical approach that focuses individuals that are facing psychotic symptoms for the first time ${ }^{12}$. At the same time that early intervention has been considered a paradigm in preventive psychiatry, there is a global, unsatisfied demand for services of this kind, which is particularly acute in Latin America. There are continuous challenges with which these services have to deal in order to properly care for large in-need populations of children, adolescents, and young adults. The objective of this study is to describe Latin America's early intervention services (EIS) in psychosis and to shed light into their challenges and particularities.

\section{Method}

In order to identify Latin American EIS, we conducted an internet-based search that included medical societies' websites, published articles, and personal contacts. We consulted the International Early Psychosis Association and Schizophrenia International Research Society, as well as the websites of national Latin American psychiatric societies. Major universities' websites were also included. To find Latin American groups that had published articles related to EIS, we consulted the databases PubMed, PsycINFO, Cochrane Library, and SciELO to look for articles about first episode and prodromal psychosis published by researchers from Latin American centers. We screened posters of the main congresses in psychosis, like the American Psychiatric Association (APA) Meeting, the International Congress on Schizophrenia Research (ICOSR), the World Psychiatric Association (WPA) Congress, and the Schizophrenia International Research Society South America Conference (SIRS). Furthermore, we made personal contacts with services' staffs to gather additional information.

\section{Results}

\section{Early intervention services in Latin America}

We identified seven programs focused on early intervention in psychosis. Most of them are offered by universities as part of professional training, research or both. There are some services exclusively focused on managing the first psychotic episode, while others present a wider scope and provide support during prodromal stages.

In the following section, we describe the main programs and research protocols.

1. First Episode Psychosis Program (PEP), Federal University of São Paulo (UNIFESP), São Paulo, Brazil

The First Episode Psychosis Program (PEP) was created in
1999 by members of the Department of Psychiatry of the Federal University of São Paulo (UNIFESP), Brazil. It is structured as an outpatient unit, with patients often been referred from psychiatric emergency services or inpatient units, especially those located within the catchment area of Hospital São Paulo, which is ran by UNIFESP.

Individuals that receive a diagnosis of first psychotic episode by members of this service are offered the opportunity to take part in the weekly sessions of a 12-week psycho-educative group and to have a clinical follow-up with a specialized psychiatrist. After that period, care continues to be provided as necessary in accordance with the patients' clinical demands, at the same time that they are invited to take part in an employment support group.

The program also develops medical and health professionals training as a part of the residence and post-graduate programs in psychiatry and medical psychology of UNIFESP. The main focus of research in PEP are metabolic syndrome in first episode patients and family interventions ${ }^{13,14}$.

\section{The ASAS Program, Universidade de São Paulo, São Paulo,} Brazil

The ASAS Program (Evaluation and Follow-up of Adolescents and Young Adults in São Paulo) is an initiative sponsored by the Institute of Psychiatry of the University of Sáo Paulo Medical School, which aims to provide care for undiagnosed, drug-naïve individuals (14-30 years old), during two consecutive years. The program started with an informative campaign that covered high schools and different media, with the objective of encouraging youngsters to seek the service's help, once a suspect of psychosis had been raised. During the same period (2004-2007), researchers of the ASAS program conducted a phone-based screening, which targeted the identification of 'at risk' youngsters who were invited to participate in a deeper screening process, where several psychiatric scales were applied. The investigators contacted 894 individuals, among which only 141 fulfilled the criteria to be enrolled in the personal assessment and 18 (2\%) were finally identified as actually pertaining to the 'at risk' group for psychosis.

3. Program for the Recognition and Early Intervention in Psychosis (PRIP), Department of Psychiatry, Universidade Federal do Rio de Janeiro, Rio de Janeiro, Brazil

This program was created in 2010 by members of the Department of Psychiatry from the Federal University of Rio de Janeiro. It provides assistance for patients in early stages of psychosis, at the same time that it fosters research activities. The group is currently translating and adapting psychiatric scales, such as the Examination of Anomalous Self-Experience (EASE), which targets schizophrenic manifestations. This scale was developed by a group of researchers affiliated to the University of Copenhagen and is inspired in the Bonn Scale of primary symptoms. In addition, members of the group organized the 1st International Symposium and Workshop on Early Recognition and Intervention on Psychosis, with the objective of disseminating knowledge and 
training professionals on the proper use of standardized psychiatric instruments.

\section{Program of Recognition and Intervention for Individuals in At-Risk Mental States (PRISMA), Federal University of São Paulo (UNIFESP), Brazil}

PRISMA is an outpatient clinic created in 2010 to identify and offer treatment to individuals presenting at-risk mental states and to promote research in the field committed to the Brazilian reality. In its first year, PRISMA was responsible for the consolidation of a multi-professional team of more than 20 professionals, which included child and adult psychiatrists, psychologists, nutritionists, physical education teachers, social workers, and others. The Comprehensive Assessment of At-Risk Mental States (CAARMS) was adopted to confirm the diagnosis and its translation and validation was the first project of the group. Up to current days, 70 individuals were interviewed, 10 of which were considered at-risk. The individuals who were considered at-risk underwent a battery of clinical tests: a risk factor questionnaire (migration, parental age, violence exposure, obstetric complications, perinatal and childhood CNS infections), complete CAARMS, Young Mania Rating Scale, schizotypal inventory (based on DSM-IV criteria), Family History Screen, and quality-of-life measure (SF-36). The aim of the study is to enroll 100 ARMS individuals for a 2-year follow-up study. The research protocol comprises the clinical assessment described above, blood samples for genotyping and study of peripheral biomarkers, neuropsychological testing, and MRI scans ${ }^{15-17}$.

5. Adolescent Program of Neuropsychiatric and Imaging Study (PIENSA), National Institute of Neurology and Neurosurgery of Mexico, Mexico City, Mexico

The National Institute of Neurology and Neurosurgery of Mexico (INNN) is located in Mexico City, a catchment area of over 22 million people, and is part of the National Institutes of Health in Mexico. In the INNN, there is a Neuropsychiatry Unit, an inpatient unit, and two outpatient services devoted to early intervention: the Adolescent Program of Neuropsychiatric and Imaging Study (PIENSA), mainly for prodromal subjects, and the First Psychotic Episode Clinic, that does the patients' follow-up when they are discharged. Studies conducted in the INNN have been published with a casuistic of 18 UHR subjects evaluated using a translated version of the Structured Interview for Prodromal Syndromes (SIPS) criteria $^{18}$ and with an equal number of individuals in their $\mathrm{FEP}^{19}$. The main strategy of research with UHR and FEP in the INNN has been neuroimaging, specifically focusing in glutamate levels in the pre-commissural dorsal-caudate and cerebellum of UHR and FEP individuals ${ }^{19}$.

\section{Research protocols for early intervention in psy- chosis}

The search also returned articles and websites describing research protocols in early intervention in psychosis that are not necessarily linked to assistance. These protocols have the objective of exploring research questions regarding high risk for psychosis.

\section{The National Institute of Developmental Psychiatry for Children} and Adolescents, São Paulo and Porto Alegre, Brazil

The National Institute of Developmental Psychiatry for Children and Adolescents (INPD) is a National Institute of Science and Technology (INCT) created and funded by CNPq and FAPESP to foster the development of knowledge on childhood mental health and disorders in Brazil. The institute is a national consortium with the University of Sáo Paulo Medical School as its home institution, and is composed of approximately 50 researchers from 10 Brazilian and international universities. The INPD research projects aim to define the "at risk status" for mental disorders in childhood, to delineate developmental trajectories, and to design early intervention programs for mental disorders. Three large cohorts have been followed-up: (1) a cohort of 900 women in their 8th month of pregnancy and their children assisted at primary care services in the Butantã region, in the metropolitan area of São Paulo. Potential factors occurring at prenatal, perinatal, and early infancy periods that may predict mental disorders will be assessed and children and their families will be followed initially up to 5 years of age. At the moment, 400 children and their mothers have already been included and are being followed; (2) a cohort of 6-12 years old scholars from 57 schools of two Brazilian capitals (São Paulo and Porto Alegre). A total of 9,937 families were screened for index-child and familial psychopathology. From this sample, 1,500 children were selected based on the risk for 5 types of psychiatric disorders (attention/deficit hyperactivity disorder, anxiety disorders, obsessive-compulsive disorder, early psychoticlike symptoms, and learning disorders) and another 1,000 were randomly chosen as controls. All subjects were evaluated for mental health and neuropsychological status and saliva samples were collected for genetic studies. In addition, a sub-sample of 750 children are performing MRI scans and providing blood samples for the measurement of biological markers; and (3) a clinical sample of children from 2 to 5 years old referred to the Institute of Psychiatry of the University of São Paulo because of emotional and behavioral problems. Investigators will study the types of early symptoms, family functioning profile, environmental and genetic risks, and neuroimaging information to predict mental disorders later in childhood. The children will be assessed every 6 months, initially for 5 years. In addition to the research projects, the INPD has been strongly committed to education and training and knowledge transference to society ${ }^{20}$.

\section{Cuca-legal Project}

The Cuca-legal [T.N.: Nice Mind] program was created by the same group of PRISMA as a joint initiative and aims to increase mental health awareness in the public educational system. Education in Brazil is regulated by the Federal Government through the Ministry of Education, and children must attend school for a minimum of nine years. As nurses and psychologists 
are rarely available in Brazilian public schools, teachers are in a strategic position. If properly trained, teachers may recognize earlier and refer children and adolescents with more severe mental health problems. Training programs for teachers resulting from a close collaboration between mental health professionals and the educational system could benefit students by promoting mental health and well-being campaigns, reducing stigma related to the help seeking process, facilitating service access, increasing treatment compliance, and preventing academic failure and dropout. Cuca-legal conducted a pilot study in a public school located in the city of São Paulo. The school had 1,500 students enrolled between fifth grade and the last year of high school. An intervention directed to the students was also assessed. Fifty referred students were evaluated, 2 of which were later identified as ARMS and are being followed in PRISMA. This first study will be extended to 10 elementary schools so as to reach about 3,000 students and over 200 teachers.

\section{Challenges for EIS and research in Latin America}

Schizophrenia is traditionally thought to have better outcomes in people with psychosis living in low- and middle-income countries $^{21}$. However, it is unclear if this notion can be applied for Latin America, as most follow-up studies in low- and middle-income countries were conducted in Asia and Africa ${ }^{22}$. Nonetheless, the better outcome idea has been increasingly challenged by evidence that schizophrenia patients in "developing" countries have a significant excess of mortality ${ }^{22,23}$. More importantly, like high income countries, DUP is also associated to poorer response to treatment and increased levels of disability in low- and middle-income countries ${ }^{24}$, highlighting that, no matter the cultural context, lack of treatment predicts worse outcome. Unfortunately, it is known that having little or no access to mental health care in Latin America is the rule, rather than the exception.

Moreover, demographic and economic changes are taking place and have the potential to affect the current situation. Migration from rural to urban centers is an ongoing phenomenon in many Latin American countries and is considered an important risk factor for psychosis ${ }^{25}$. Another consequence is the disorganization of urban centers, which may lead to situations of poverty, social disadvantage and inequality that are also considered significant causes in the set of etiologic factors of psychosis ${ }^{26}$.

As resources in developing countries are frequently insufficient, they need to be used in the most efficient way so as to benefit the highest number of individuals. While most of the studies that were conducted to evaluate cost-effectiveness demonstrate that EIS actually cost less than usual care ${ }^{27,28}$, some have suggested that the costs of both approaches are about the same ${ }^{29}$. The difference is mainly due to an expressive reduction in inpatient intervention costs in the EIS group. Combining this information with the consistent data of better outcomes in early treated patients $s^{30,31}$ leads to the conclusion that EIS not only are beneficial to patients but also tend to be cost-effective.

Cost-effectiveness analyses concerning individuals in at risk mental states have been conducted in only two studies. The first of them, from Melbourne, Australia, demonstrated that, compared with standard care, their specialized preventive program is cost effective. While patients receiving preventive care cost an average of $A \$ 3,445$ per year, patients in treatment as usual cost about $A \$$ 9,503 per year ${ }^{28}$. In a 12 -month analysis of the cost-effectiveness of the Outreach and Support in South London (OASIS) program, the cost of the intervention was $£ 1,872$ higher than conventional care; however, after 24 months, net costs were $£ 961$ less than in the conventional care group, since other factors like unemployment, for example, were less probable in the OASIS gCurrently, the estimated number of individuals with schizophrenia living in Latin America exceeds 5 million. Latin America has a population 28 times bigger than Australia, and while Australia has 25 EIS, only 7 such centers in Latin America were found by this search. There is a dramatic contrast between Latin American countries and Canada (40 centers), the United Kingdom (17 centers), the United States (18 centers), and Germany (5 centers).

Some issues are particularly important regarding EIS in Latin America. As people who do not know their health status or the risks to it potentially constitute a major source of unmet needs in countries where manifestations of mental disorders are frequently interpreted according to cultural biases, they are not considered to require medical attention. It is a challenge to all services described here to inform and educate professionals and the general public to find ways of reaching this population.

With no exception, all the centers found are located in big cities, most of them being part of tertiary hospitals or universities. Distance and time can be crucial for the outcome of medical emergencies. Since first episode psychosis and possibly prodromal psychosis are true emergencies, the lack of EIS in rural areas or in distant cities can be a problem.

Collaboration between clinical and research teams should be implemented to scale up the number of patients assisted, increase follow-up time, assess predictors of conversion to psychosis in ARMS individuals, establish more valid diagnostic categories for FEP, and produce and implement therapeutic guidelines adapted to local conditions. A map of EIS in Latin America could be useful for clinicians and researchers to understand the similarities and differences between these services and those in developed countries.

\section{Conclusion}

The importance of early intervention in psychotic disorders is well documented and is established nowadays as one of the best ways to care for patients with severe mental disorders. EIS provide patients with assistance in a crucial period of the disease, thus improving quality of life and prognosis, while allowing the implementation of preventive strategies. From a public health perspective, EIS are also relevant as proven cost-effective initiatives. Therefore, no mental health system should lack such valuable services. As shown here, Latin American countries are deeply deficient in terms of specialized EIS. Moreover, Latin American research in the area is still in its early days, leaving unmet the 
demand for regionally adapted knowledge that could upgrade patient care and service planning. In this context, investments and further integration with clinicians and researchers from different countries are mandatory in order to improve the situation.

\section{Acknowledgements}

The authors are thankful to Dr. Camilo de la Fuente-Sandoval from the Experimental Psychiatry Laboratory of the Instituto Nacional de Neurologia y Neurocirurgia, Mexico City, Mexico; to Professor Jair de Jesus Mari, from the Department of Psychiatry of the Universidade Federal de São Paulo; and to Dr. Nelson Goldenstein from the Institute of Psychiatry (IPUB) of the Universidade Federal do Rio de Janeiro.

Disclosures and Role of Funding Sources

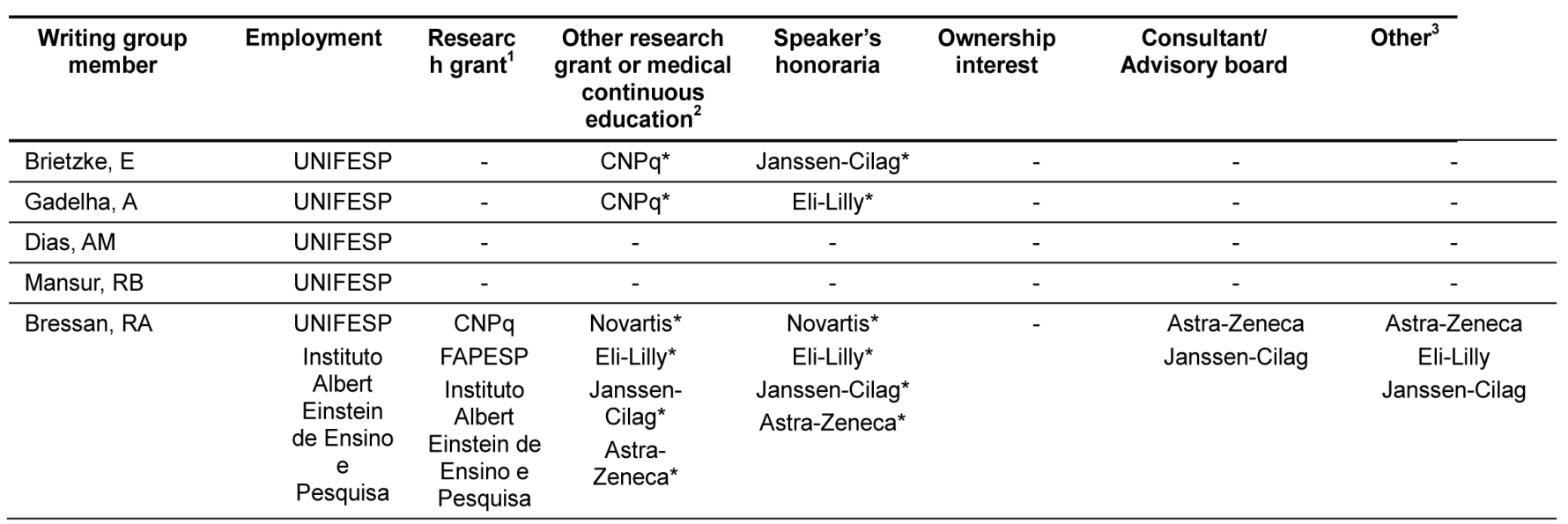

* Modest

** Significant

*** Significant: Amounts given to the author's institution or to a colleague for research in which the author has participation, not directly to the author.

\section{References}

1. National Institute of Mental Health. Towards a Model for a Comprehensive Community-Based Mental Health System. Washington, DC: NIMH; 1987.

2. Menezes PR, Scazufca M, Busatto GF, Coutinho LMS, Mcguire PK,,Murray RM. Incidence of first-contact psychosis in São Paulo, Brazil. Br J Psychiatry. 2007;191(51):S102-106

3. Maier W, Cornblatt BA, Merikangas K.R. Transition to schizophrenia and related disorders: toward taxonomy of risk. Schizophr Bull. 2003;29(4): 693-701.

4. Nordentoft M, Jeppesen P, Petersen L, Bertelsen M, Thorup A. The rationale for early intervention in schizophrenia and related disorders. Early Interv Psychiatry. 2009;3 Suppl 1:S3-7.

5. Knapp M, Mangalore R, Simon J. The global costs of schizophrenia. Schizophr Bull. 2004;30(2): 279-293.

6. Edwards J, Harris MG, Bapat S. Developing services for first-episode psychosis and the critical period. Br J Psychiatry. 2005;187(48):S91-97.

7. Nielssen O, Large M. Rates of homicide during the first episode of psychosis and after treatment: a systematic review and meta-analysis. Schizophr Bull. 2010;36(4):702-12.

8. Rinaldi M, Killackey E, Smith J, Shepherd G, Singh SP, Craig T. First episode psychosis and employment: a review. Int Rev Psychiatry. 2010;22(2):148-62.

9. Upthegrove R, Birchwood M, Ross K, Brunett K, McCollum R, Jones L. The evolution of depression and suicidality in first episode psychosis. Acta Psychiatr Scand. 2010;122(3):211-8.

10. Archie S, Rush BR, Akhtar-Danesh N, Norman R, Malla A, Roy P, Zipursky $R B$. Substance use and abuse in first-episode psychosis: prevalence before and after early intervention. Schizophr Bull. 2007;33(6):1354-63.

11. Perkins DO, Gu H, Boteva K, Lieberman JA. Relationship between duration of untreated psychosis and outcome in first-episode schizophrenia: A critical review and meta-analysis. Am J Psychiatry. 2005;162(10):1785-1804.
12. Singh SP. Early intervention in psychosis. BrJ Psychiatry. 2010;196(5):343-345. 13. Cabral RR, Chaves AC. Multi-family group intervention in a programme for patients with first-episode psychosis: a Brazilian experience. Int J Soc Psychiatry. 2010;56(5):527-32.

14. Attux C, Quintana MI, Chaves AC. Weight gain, dyslipidemia and altered parameters for metabolic syndrome on first episode psychotic patients after sixmonth follow-up. Rev Bras Psiquiatr. 2007;29(4):346-9.

15. Gadelha A, Silva PFR, Melcop AC, Pan Neto PM, Moriyama TS, GraeffMartins AS, Bordin IA, Bressan RA. The PRISMA clinic - A Brazilian initiative for detection and follow-up of individuals at risk for psychosis - Description of the clinical protocol. 7th biennial conference of the International Early Psychosis Association - 29th November to 1st December 2010, Amsterdan.

16. Silva PFR, Gadelha A, Melcop AC, Pan Neto PM, Moriyama TS, GraeffMartins AS, Bordin IA, Bressan RA. Validation of the Portuguese version of the Comprehensive Assessment of At-Risk Mental States (CAARMS). 7th biennial conference of the International Early Psychosis Association - 29th November to 1st December 2010, Amsterdan.

17. Melcop AC, Gadelha A, Silva PFR, Pan Neto PM, Moriyama TS, GraeffMartins AS, Bordin I, Bressan RA. The Portuguese version of the Y-PARQ as a screening instrument in prodromal research. 2010. 7th biennial conference of the International Early Psychosis Association - 29th November to 1st December 2010, Amsterdan.

18. Miller TJ, McGlashan TH, Rosen JL, Cadenhead K, Cannon T, Ventura J, McFarlane W, Perkins DO, Pearlson GD, Woods SW. Prodromal assessment with the structured interview for prodromal syndromes and the scale of prodromal symptoms: predictive validity, interrater reliability, and training to reliability. Schizophr Bull. 2003;29(4):703-15.

19. De La Fuente-Sandával C, León-Ortiz P, Favila R, Stephano S, Mamo D, Ramírez-Bermúdez J, Graff-Guerrero A. Higher levels of glutamate in the associativestriatum of subjects with prodromal symptoms of schizophrenia and patients with first-episode psychosis. Neuropsychopharmacology. 2011;36(9):1781-1791. 
20. Miguel EC, Mercadante MT, Grisi S, Rohde LA. The National Science and Technology Institute in Child and Adolescence Developmental Psychiatry: a new paradigm for Brazilian psychiatry focused on our children and their future. Rev Bras Psiquiatr. 2009;31(2):85-8.

21. Jablensky A, Sartorius N, Ernberg G, Anker M, Korten A, Cooper JE, Day $\mathrm{R}$, Bertelsen A. Schizophrenia: manifestations, incidence and course in different cultures. A World Health Organization ten-country study. Psychol Med Monogr Suppl. 1992;20:1-97.

22. Cohen A, Patel V, Thara R, Gureje O. Questioning an axiom: better prognosis for schizophrenia in the developing world? Schizophr Bull. 2008;34(2):229-44.

23. Ran MS, Chen EY, Conwell Y, Chan CL, Yip PS, Xiang MZ, Caine ED. Mortality in people with schizophrenia in rural China: 10-year cohort study. $\mathrm{Br}$ J Psychiatry. 2007;190:237-242.

24. Farooq S, Large M, Nielssen O, Waheed W. The relationship between the duration of untreated psychosis and outcome in low-and-middle income countries: a systematic review and meta-analysis. Schizophr Res. 2009;109(1-3):15-23.

25. Krabbendam L, van Os J. Schizophrenia and urbanicity: a major environmental influence--conditional on genetic risk. Schizophr Bull. 2005;31(4):795-9.

26. Patel V, Flisher AJ, Hetrick S, McGorry P. Mental health for young people: a global public health challenge. Lancet. 2007;369(9569):1302-1313.

27. Angelo C, Vittorio M, Anna M, Antonio P. Cost-effectiveness of treating firstepisode psychosis: five-year follow-up results from an Italian early intervention programme. Early Interv Psychiatry. 2011;5(3):203-11.

28. Mihalopoulos C, Harris M, Henry L, Harrigan S, McGorry P. Is early intervention in psychosis cost-effective over the long term? Schizophrenia Bull. 2009;35(5):909-918.

29. McCrone P, Craig TK, Power P, Garety PA. Cost-effectiveness of an early intervention service for people with psychosis. Br J Psychiatry. 2010;196(5):377-82.

30. Marshall M, Lewis S, Lockwood A, Drake R, Jones P, Croudace T. Association between duration of untreated psychosis and outcome in cohorts of first-episode patients: a systematic review. Arch Gen Psychiatry. 2005;62(9):975-83.

31. Perkins DO, Gu H, Boteva K, Lieberman JA. Relationship between duration of untreated psychosis and outcome in first-episode schizophrenia: a critical review and meta-analysis. Am J Psychiatry. 2005;162(10):1785-804.

32. Valmaggia LR, McCrone P, Knapp M, Woolley JB, Broome MR, Tabaham P, Johns LC, Prescott C, Bramon E, Lappin J, Power P, McGuire P. Economic impact of early intervention in people with at risk states for psychosis. Psychol Med. 2009;39(10):1617-1626. 